Gefässchirurgie 2021 · 26:469-472

https://doi.org/10.1007/s00772-021-00789-7

Angenommen: 3. Juni 2021

Online publiziert: 5 . Juli 2021

(c) Der/die Autor(en) 2021

\section{Zum aktuellen Stand von transcarotidaler arterieller Revaskularisation (TCAR)}

\author{
Martin Storck ${ }^{1} \cdot$ Reinhart T. Grundmann ${ }^{2,3}$ \\ 'Gefäßzentrum Karlsruhe, Klinik für Gefäß- und Thoraxchirurgie, Städt. Klinikum Karlsruhe, Karlsruhe, \\ Deutschland \\ ${ }^{2}$ Universitäres Herz- und Gefäßzentrum UKE Hamburg GmbH, Universitätsklinikum Hamburg-Eppendorf, \\ Hamburg, Deutschland \\ ${ }^{3}$ Deutsches Institut für Gefäßmedizinische Gesundheitsforschung (DIGG) der Deutschen Gesellschaft für \\ Gefäßchirurgie und Gefäßmedizin, Berlin, Deutschland
}

Die transcarotidale arterielle Revaskularisation (TCAR) - Carotisstenting über einen zervikalen Zugang mit dem ENROUTE Transcarotid Neuroprotection and Stent System, technische Details bei [10] ist ein Ansatz, die Sicherheit der endovaskulären Carotisrevaskularisation zu erhöhen. Auf diese Weise können eine Femoraliskanülierung und Navigation durch die Aorta und den Aortenbogen vermieden werden. Die TCAR bietet darüber hinaus einen Neuroprotektionsmechanismus durch eine vorübergehende Umkehr des cerebralen Flusses. In der multizentrischen ROADSTER-2-Studie [6] wurde das Verfahren bei 632 Patienten angewandt, mit überzeugenden periprozeduralen Ergebnissen: 1 Patient starb $(0,2 \%)$, es gab 4 Schlaganfälle $(0,6 \%)$ und 6 Herzinfarkte $(0,9 \%)$. Die Rate für den Kompositendpunkt Schlaganfall/Tod/ Herzinfarkt wurde mit 1,7\% angegeben. In diese Studie waren 467 asymptomatische und 165 symptomatische Patienten eingeschlossen worden. Die Autoren sahen das Verfahren speziell für Hochrisikopatienten als indiziert an und empfahlen es aufgrund der möglichen Reduzierung cerebraler Embolisationen im Vergleich zum transfemoralen Carotisstenting (TFCAS). In der vorliegenden Arbeit soll der aktuelle Studienstand der letzten zwei Jahre im Rahmen unserer Reihe zur evidenzbasierten Gefäßchirurgie dargestellt werden. Grundlage war eine Pubmed-Recherche unter dem Schlüsselwörtern "transcarotid artery revascularization".

\section{Metaanalysen}

Es liegen mittlerweile drei Metaanalysen mit systematischem Review vor. Naazie et al. [11] verglichen TCAR mit Flussumkehr mit dem transfemoralen Carotisstenting (TFCAS) und der Carotisendarteriektomie (CEA). Sie fanden 9 nichtrandomisierte Studien mit 4012 Patienten, bei denen TCAR vorgenommen wurde. Das 30-Tage-Risiko nach TCAR betrug über alle für Schlaganfall/Tod 1,89\%, Schlaganfall $1,34 \%$, Tod $0,76 \%$, Herzinfarkt $0,6 \%$, Schlaganfall/Tod/Herzinfarkt 2,2\% und Hirnnervenschädigung 0,31\%. Zwei nichtrandomisierte Studien gaben an, dass TCAR mit einem niedrigeren Risiko für Schlaganfall und Tod assoziiert ist im Vergleich zu TFCAS. Im Vergleich zu CEA ließen 4 nichtrandomisierte Studien ein geringeres Risiko für Hirnnervenschädigungen bei TCAR vermuten, aber kein signifikant unterschiedliches Risiko für Schlaganfall, Schlaganfall/Tod und Schlaganfall/Tod/ Herzinfarkt. Der Vorteil von TCAR vor der CEA läge demnach allein in der geringeren Rate an Hirnnervenschädigungen.

Zwei weitere Metaanalysen wurden anschließend publiziert. Die Metaanalyse von Galyfos et al. [5] basiert auf 18 klinischen Studien mit insgesamt 8473 Patienten, davon 4852 mit TCAR. Die gepoolte 30Tage-Sterblichkeit machte $0,7 \%$ aus, die Schlaganfall/TIA(transitorische ischämische Attacke)-Rate 2,0\% und die Rate an frühem Herzinfarkt 0,4\%. Die technische Erfolgsrate wurde mit 97,6\% angegeben, die Rate an Hirnnervenschädigungen mit 1,2\%. Die Restenoserate nach 3 bis 40 Monaten Follow-up war 4\%. Die Autoren betonten die niedrige Sterblichkeitsund Schlaganfallrate und Rate an Hirnnervenschädigungen, wiesen aber auch darauf hin, dass symptomatische Patienten ein höheres perioperatives Risiko zu haben scheinen. Inwieweit TCAR einer CEA gleichwertig, über- oder unterlegen ist, ließ sich aufgrund des Mangels an Vergleichsstudien mit Langzeitergebnissen nicht feststellen.

Die dritte Metaanalyse von Sagris et al. [13] fußt auf 49 Studien mit 14.588 Patienten. Auch diese Autoren betonten die Sicherheit von TCAR (periprozedurale Sterblichkeit jeglicher Ursache 0,5\%, Schlaganfallrate 1,3\%). Sie nannten eine technische Erfolgsrate von $99 \%$ und wiesen auf die sehr niedrige Rate an Hirnnervenschädigungen (bei 33 von 8994 Patienten) hin. Ein Vergleich mit CEA und CAS (Carotisstenting) konnte allerdings auch in dieser Metaanalyse nicht vorgenommen werden, da die meisten der inkludierten Studien einarmig waren und eine gepolte Synthese der Daten nur für das 30-Tage-Ergebnis machbar war.

\section{Studien}

TCAR vs. transcarotidales Stenting mit distaler Embolieprotektion (TCAS-DEP)

Über die Ergebnisse mit TCAR berichteten Naazie et al. [12] anhand des Registers der Vascular Quality Initiative (VQI) der Jahre 
2016 bis 2019. Sie überprüften den Nutzen der Flussumkehr im Vergleich zur distalen Embolieprotektion (DEP) bei transcarotidalem Stenting (TCAS). Bei 8462 Patienten mit TCAR wurde in 8139 (90,6\%) Fällen mit Flussumkehr vorgegangen, bei 287 (3,4\%) wurde eine distale Embolieprotektion eingesetzt (TCAS-DEP). In einer Matched-pairAnalyse (274 Paare) war das Vorgehen unter Flussumkehr nach 30 Tagen mit einem signifikant niedrigeren Risiko für Schlaganfall/Tod $(1,8 \%$ vs. $5,5 \%)$ und Schlaganfall $(0,7 \%$ vs. $3,7 \%)$ assoziiert, verglichen mit TCAS unter distaler Embolieprotektion. Die Unterschiede für den Kompositendpunkt Schlaganfall/Tod/Herzinfarkt nach 30 Tagen $(2,6 \%$ vs. $5,5 \%)$ waren allerdings statistisch nicht signifikant $(p<0,088)$. Ebenso unterschieden sich beide Gruppen nicht in der Sterblichkeit nach 30 Tagen (1,5\% vs. $1,8 \%)$. Die Botschaft dieser Untersuchung war, dass die dynamische Flussumkehr bei TCAR eine bessere Neuroprotektion bietet als distale Embolieprotektionsfilter bei transcarotidalem Vorgehen. Darüber hinaus konnten die Autoren zeigen, dass die Vermeidung des Aortenbogens beim CAS zu keiner Reduktion der Schlaganfallrate führt (transcarotidales Stenting mit Embolieprotektion vs. transfemorales Stenting mit Embolieprotektion: Schlaganfall/Tod $4,9 \%$ vs. 3,7\%; $p=0,65$ ). Nach dieser Untersuchung scheint TCAR bessere Ergebnisse aufzuweisen als das herkömmliche transfemorale CAS mit distaler Filterprotektion. Die bessere Neuroprotektion beruht auf der Flussumkehr. Der Vermeidung des Aortenbogens beim CAS kommt nicht die entscheidende Bedeutung zu, um speziell die Schlaganfallrate nach CAS zu senken.

\section{TCAR vs. transfemorales Carotisstenting (TFCAS)}

Malas et al. [9] identifizierten in der Datenbank der VQI 10.136 Patienten mit TFCAS und stellten sie 638 Patienten mit TCAR gegenüber. Patienten mit TCAR waren signifikant älter und hatten eine höhere kardiale Komorbidität, waren aber häufiger asymptomatisch und hatten seltener eine Rezidivstenose. Die Raten an Krankenhaus-Schlaganfallrate/TIA und TIA/ Schlaganfall/Tod waren nach TFCAS signifikant höher als nach TCAR (3,3\% vs. 1,9\% und $3,8 \%$ vs. $2,2 \% ; p=0,04)$. Nach beiden Verfahren hatten symptomatische Patienten höhere Raten an TIA/Schlaganfall/Tod als asymptomatische. In der multivariablen Analyse unterschieden sich TFCAS und TCAR in der Rate an Schlaganfall/Tod nur im Trend (2,5\% vs. $1,7 \% ; p=0,25)$. Jedoch war TFCAS mit der doppelten Odds an unerwünschten neurologischen Ereignissen und TIA/Schlaganfall/Tod im Vergleich zu TCAR assoziiert, unabhängig vom Symptomstatus der Patienten. Die Daten belegten erstmals in einer flächendeckenden Erhebung Vorteile von TCAR gegenüber TFCAS.

Liang et al. [8] identifizierten in der VQI-Carotis-Stenting-Database der Jahre 2005 bis 2019 insgesamt 24.232 Patienten mit CAS. Es wurden 561 Eingriffe (2,3\%) mit TFCAS unter proximaler Ballonokklusion vorgenommen, 18.126 (74\%) mittels TFCAS unter distaler Embolieprotektion und 5545 (22,9\%) mit TCAR unter Flussumkehr. Mittels „propensity matching“ wurden zwei vergleichbare Gruppen gebildet (463 Paare), die entweder mit TFCAS unter Schutz eines Embolieprotektionssystems oder mit TFCAS unter proximaler Ballonokklusion behandelt wurden. Die Krankenhaus-Schlaganfall/Sterblichkeitsrate war bei TFCAS mit proximaler Ballonokklusion 3,2\%, unter TFCAS mit distaler Embolieprotektion 3,7\%, ein statistisch nicht signifikanter Unterschied. Ebenso waren Schlaganfallrate $(2,4 \%$ vs. $2,6 \%)$, TIA, Tod und Herzinfarkt nicht unterschiedlich. Jedoch war TFCAS mit proximaler Ballonokklusion mit einer höheren Rate an hämodynamischer hypotensiver Instabilität assoziiert ( $19 \%$ vs. $14 \%, p=0,03$ ) und der Verbrauch an Kontrastmittel war signifikant geringer. In einem weiteren Schritt wurden Propensity-gematcht 357 Paare mit TFCAS und Ballonokklusion vs. TCAR unter Flussumkehr gebildet. In diesem Vergleich war TFCAS mit proximaler Ballonokklusion mit einer signifikant höheren Krankenhaus-Schlaganfallrate oder Tod assoziiert, verglichen mit TCAR unter Flussumkehr (3,1\% vs. 0.8\%; RR, 3,67; $95 \%$ $\mathrm{Cl}, 1,02-13,14 ; p=0,03)$. Im Trend waren auch Tod $(0,8 \%$ vs. $0,0 \%)$ und Schlaganfall (2,5\% vs. $0,8 \%)$ bei TFCAS höher. Im Endeffekt hatten demnach TFCAS mit distaler Embolieprotektion oder proximaler Ballonokklusion ähnliche Raten an
Schlaganfall/Tod, während TCAR mit Flussumkehr dem TFCAS mit Ballonokklusion signifikant überlegen war. Das Fazit war: Das transfemorale CAS mit proximaler Ballonokklusion bietet nicht den gleichen Grad an Embolieprotektion wie TCAR mit Flussumkehr.

\section{TCAR vs. CEA}

Anhand der retrospektiven Daten von 4 Zentren stellten Kashyap et al. [7] einen Propensity-Score gematchten Vergleich von TCAR (benutzt wurde das ENROUTE Transcarotid Neuroprotection System) vs. CEA an. In die Untersuchung gingen jeweils 292 Eingriffe ein. Die 30-TageSchlaganfallrate ( $1 \%$ vs. $0,3 \%$ ) und Tod ( $0,3 \%$ vs. $0,7 \%)$ sowie Schlaganfall $(2,8 \%$ vs. $2,2 \%)$ und Tod (1,8\% vs. $4,5 \%)$ nach 1 Jahr unterschieden sich nicht signifikant, jedoch war die Rate an Hirnnervenschädigungen bei TCAR signifikant geringer $(0,3 \%$ vs. $3,8 \%, p=0,01)$. Die Autoren betonten, dass dieselben Chirurgen in dergleichen Institution mit TCAR und CEA vergleichbare Ergebnisse erzielten, bei reduziertem Risiko für eine Hirnnervenschädigung unter TCAR. Inwieweit diese Ergebnisse, die in erfahrenen Zentren erzielt wurden, generell übertragbar sind, ließen die Autoren offen. Eine retrospektive Monocenterstudie, in der 66 Patienten mit TCAR 501 Patienten mit CEA gegenübergestellt wurden, kam ebenfalls zu der Schlussfolgerung, dass TCAR ein sicheres Verfahren darstellt. In dieser Erhebung waren prozedurbezogene Schlaganfallrate und perioperative Komplikationen (einschließlich Herzinfarkt, Tod, Hirnnervenschädigung und Hämatome) den Raten nach CEA in allem vergleichbar [1].

Von Januar 2016 bis März 2018 wurden im Register der VQI 1182 Patienten mit TCAR und 10.797 Patienten mit CEA erfasst. Für TCAR wurde das ENROUTE Transcarotid Neuroprotection System for flow reversal genutzt. Beide Vorgehensweisen wurden von Schermerhorn et al. [14] miteinander verglichen. Patienten mit TCAR waren älter (median 74 vs. 71 Jahre; $p<0,001$ ), waren häufiger symptomatisch ( $32 \%$ vs. $27 \%$; $p<0,001)$ und hatten die höhere Komorbidität. Nicht adjustiert ergaben sich keine signifikanten Unterschiede zwischen TCAR und CEA hinsichtlich des Kompositend- 
punkts Schlaganfall/Tod (1,6\% vs. $1,4 \%)$, Schlaganfall allein $(1,4 \%$ vs. $1,2 \%)$ oder hinsichtlich Schlaganfall/Tod/Herzinfarkt $(2,5 \%$ vs. $1,9 \%)$. Auch bei Patienten mit kontralateralem Verschluss fanden sich keine signifikanten Unterschiede bei postoperativem Schlaganfall $(0,0 \%$ vs. 1,4\%). Die TCAR war mit einer signifikant geringeren Rate an Hirnnervenschädigungen $(0,6 \%$ vs. $1,8 \% ; p<0,001)$ und kürzerer Operationszeit (78 vs. $111 \mathrm{~min}$; $p<0,001)$ assoziiert. Eine postoperative hypertensive Instabilität war nach TCAR seltener ( $10 \%$ vs. $21 \% ; p<0,001)$, eine hypotensive Instabilität jedoch häufiger (13\% vs. $9,8 \% ; p<0,01)$. Hinsichtlich eines Reperfusionssyndroms unterschieden sich beide Gruppen nicht (0,3\% vs. $0,1 \%$ ). In der adjustierten Analyse ergaben sich ebenfalls keine signifikanten Unterschiede bezüglich Schlaganfall/Tod, Schlaganfall/Tod/Herzinfarkt oder in den Einzelparametern zwischen TCAR und CEA. Allerdings waren im Trend die unerwünschten Ereignisse mit Ausnahme der Krankenhaussterblichkeit bei TCAR etwas höher. Insgesamt war TCAR bei Behandlung der Carotisstenose mit ähnlichen Raten an perioperativem Schlaganfall, Tod und Herzinfarkt verbunden, verglichen mit dem Goldstandard CEA. Dies traf für symptomatische und asymptomatische Stenosen zu und für CarotisWiederholungseingriffe. Der Vorteil von TCAR lag in der kürzeren Operationszeit und den geringeren Hirnnervenschädigungen. So erwies sich TCAR als eine sichere Alternative zu CEA - mit der Einschränkung, dass längere Follow-upDaten fehlen und die Behandlungszahlen insgesamt noch klein sind.

\section{CEA, TFCAS und TCAR beim älteren Patienten}

Bisher wurde angenommen, dass beim älteren Patienten die CEA vor dem transfemoralen Carotisstenting (TFCAS) aufgrund einer geringeren postprozeduralen Schlaganfallrate Vorteile besitzt. Inwieweit dies auch für TCAR zutrifft, untersuchten Dakour-Aridi et al. [4] in der VQI-Datenbasis der Jahre 2015 bis 2018. Bei der Studienkohorte handelte es sich um 3152 TCAR, 10.381 TFCAS und 61.650 CEA. Die Ergebnisse von TCAR änderten sich mit dem Alter der Patienten nicht: Krankenhaus-Schlaganfallrate/Tod 1,4, 1,9 und $1,5 \%$ bei Patienten $<70$ Jahre, 71-79 Jahre und > 80 Jahre. Des Weiteren waren die Ergebnisse von TCAR und CEA nicht signifikant unterschiedlich. Hingegen war TCAR dem TFCAS bei Patienten > 80 Jahre signifikant überlegen, mit einer relativen Risikoreduktion von $72 \%$ beim Schlaganfallrisiko (4,7\% vs. $1 \%$ ), von $65 \%$ bei Schlaganfall/Tod $(4,6$ vs. $1,5 \%)$ und $76 \%$ bei Schlaganfall/Tod/Herzinfarkt $\quad(5,5 \%$ vs. 2,5\%). Die Botschaft war, dass TCAR ein relativ sicheres Verfahren darstellt, unabhängig vom Alter der Patienten. Der Vorteil gegenüber TFCAS kommt speziell bei Patienten $>77$ Jahre zum Tragen, unabhängig vom Symptomstatus und zusätzlicher Komorbidität. Bei älteren Patienten mit hohem chirurgischen Risiko sollte TCAR TFCAS vorgezogen werden.

\section{Zeitpunkt von TCAR bei symptomatischen Patienten}

Zu dem Zeitpunkt, an dem bei symptomatischen Patienten TCAR vorgenommen werden kann/sollte, gibt es bisher keine Untersuchung. Cui et al. [3] haben anhand der VQI-Datenbank erstmals KrankenhausSchlaganfallrate, Tod und Herzinfarkt sowie die 1-Jahres-Raten von ipsilateralem Schlaganfall und Tod bei Patienten mit TCAR untersucht, die zu unterschiedlichen Intervallen nach Beginn der Symptomatik behandelt wurden. Unterschieden wurde zwischen dringlicher (0-2 Tage), früher (3-14 Tage) und später Intervention (15-180 Tage) nach dem akuten Ereignis. Es gingen 2608 symptomatische Patienten in die Untersuchung ein. Insgesamt erfolgten $5 \%$ der Eingriffe dringlich, 36\% früh und $59 \%$ spät. Die dringliche TCAR innerhalb $48 \mathrm{~h}$ nach Symptombeginn resultierte in einer dreifach gesteigerten Odds für Schlaganfall/Tod, hauptsächlich wegen einem größeren Schlaganfallrisiko; frühe und späte Intervention unterschieden sich nicht. Nach dem Ein-Jahres-Follow-up hatten dringliche und frühe Intervention keinen Vorteil hinsichtlich Schlaganfall oder Sterblichkeit im Vergleich zur späten Intervention. Der frühe Eingriff innerhalb von 3-14 Tagen erwies sich nach Meinung der Autoren als das möglicherweise zu bevorzugende Vorgehen, da in dieser Grup- pe die Rate an Schlaganfall/Tod der bei später TCAR vergleichbar war (bei erhöhter Rate an Entlassungen in nichthäusliche Umgebung). Letztlich konnte aber lediglich gesichert werden, dass TCAR innerhalb $48 \mathrm{~h}$ nach dem akuten Ereignisse nach diesen Ergebnissen nicht empfohlen werden kann.

\section{TCAR und Zentrumserfahrung}

Columbo et al. [2] gingen von der Hypothese aus, dass TCAR speziell dann indiziert ist, wenn die CEA technisch schwierig ist (wie bei hoher Carotisbifurkation oder Läsionen nahe der Schädelbasis). Wenn dies so wäre, müssten in Zentren, die TCAR vorhalten, unter Umständen die Ergebnisse der Carotisrevaskularisation insgesamt (TCAR und CEA kombiniert) besser sein als in Zentren, wo TCAR nicht zur Verfügung steht. In ihre vergleichende Effektivitätsstudie gingen 7664 Patienten mit TCAR und 78.363 Patienten mit CEA ein, behandelt in 469 nordamerikanischen Zentren (VQI-Register). Die Zahl der Zentren, die beide Verfahren durchführten, stieg (16fach) von 15 Zentren im Jahr 2015 auf 247 Zentren im Jahr 2018 an. Gleichzeitig nahm der Anteil von TCAR an allen Carotisrevaskularisationen von 90 von 12.276 $(0,7 \%)$ auf 2718 von 15.956 (17,0\%) um das 24-Fache zu. Zentren, die TCAR durchführten, hatten über alle Carotisrevaskularisationen eine Abnahme um 10\% in der Wahrscheinlichkeit von MACE („Major Adverse Cardiovascular Event ${ }^{\prime \prime}$ ) innerhalb 12 Monaten nach TCAR-Einführung zu verzeichnen im Vergleich zu Zentren, die allein CEA vorhielten. Dabei waren die periprozeduralen Ergebnisse nach TCAR und CEA nicht unterschiedlich. Die Daten ließen vermuten, dass die Etablierung von TCAR für selektierte Fälle mit einer Verbesserung des perioperativen Outcomes nach Carotisrevaskularisation insgesamt assoziiert ist.

\section{Fazit für die Praxis}

Die Ergebnisse - vor allem des VQI(„Vacular Quality Initiative")-Registers - belegen, dass TCAR (transcarotidale arterielle Revaskularisation) eine sichere und effektive Alternative zur CEA (Carotisendarteriektomie) darstellen könnte, gleichermaßen bei symptomatischen und asymptomatischen Patienten mit Carotisstenose. Jedoch sind Vergleichsstu- 
dien mit größeren Fallzahlen und längerem Follow-up notwendig, um diese Aussage zu erhärten. Das transfemorale CAS (Carotisstenting) mit proximaler Ballonokklusion bietet nicht den gleichen Grad an Embolieprotektion wie TCAR mit Flussumkehr. Die TCAR scheint bessere Ergebnisse aufzuweisen als das herkömmliche transfemorale CAS mit distaler Embolieprotektion. Die bessere Neuroprotektion beruht auf der Flussumkehr und der Vermeidung der endovaskulären Manipulation innerhalb des Aortenbogens.

Funding. Open Access funding enabled and organized by Projekt DEAL.

\section{Einhaltung ethischer Richtlinien}

Interessenkonflikt. M. Storck und R.T. Grundmann geben an, dass kein Interessenkonflikt besteht.

Für diesen Beitrag wurden von den Autoren keine Studien an Menschen oder Tieren durchgeführt. Für die aufgeführten Studien gelten die jeweils dort angegebenen ethischen Richtlinien.

Korrespondenzadresse

Prof. Dr. Reinhart T. Grundmann

Universitäres Herz- und Gefäßzentrum UKE Hamburg $\mathrm{GmbH}$, Universitätsklinikum Hamburg-Eppendorf

Martinistr. 52, 20246 Hamburg, Deutschland r.grundmann@uke.de

Open Access. Dieser Artikel wird unter der Creative Commons Namensnennung 4.0 International Lizenz veröffentlicht, welche die Nutzung, Vervielfältigung, Bearbeitung, Verbreitung und Wiedergabe in jeglichem Medium und Format erlaubt, sofern Sie den/die ursprünglichen Autor(en) und die Quelle ordnungsgemäß nennen, einen Link zur Creative Commons Lizenz beifügen und angeben, ob Änderungen vorgenommen wurden.

Die in diesem Artikel enthaltenen Bilder und sonstiges Drittmaterial unterliegen ebenfalls der genannten Creative Commons Lizenz, sofern sich aus der Abbildungslegende nichts anderes ergibt. Sofern das betreffende Material nicht unter der genannten Creative Commons Lizenz steht und die betreffende Handlung nicht nach gesetzlichen Vorschriften erlaubt ist, ist für die oben aufgeführten Weiterverwendungen des Materials die Einwilligung des jeweiligen Rechteinhabers einzuholen.

Weitere Details zur Lizenz entnehmen Sie bitte der Lizenzinformation auf http://creativecommons.org/ licenses/by/4.0/deed.de.

\section{Literatur}

1. Cappellini CA, Zheng $H$, Lamb KM, Sooppan $R$, Coffey J, Luo RQ (2021) Outcomes of transcarotid artery revascularization and carotid endarterectomy at a single institution. Ann Vasc Surg 73:329-335. https://doi.org/10.1016/j.avsg.2020. 10.023

2. Columbo JA, Martinez-Camblor P, O'Malley AJ, Stone DH, Kashyap VS, Powell RJ, Schermerhorn ML, Malas M, Nolan BW, Goodney PP (2021) Association of adoption of transcarotidartery revascularization with center-level perioperative outcomes. JAMA Netw Open 4:e2037885

3. Cui CL, Dakour-Aridi H, Eldrup-Jorgensen J, Schermerhorn ML, Siracuse JJ, Malas MB (2021) Effects of timing on in-hospital and one-year outcomes after transcarotid artery revascularization. J Vasc Surg 73:1649-1657

4. Dakour-Aridi H, Kashyap VS, Wang GJ, EldrupJorgensen J, Schermerhorn ML, Malas MB (2020) The impact of age on in-hospital outcomes after transcarotid artery revascularization, transfemoral carotid artery stenting, and carotid endarterectomy. JVasc Surg 72:931-942

5. Galyfos GC, Tsoutsas I, Konstantopoulos T, Galanopoulos G, Sigala F, Filis K, Papavassiliou V (2021) Editor's choice-Early and late outcomes after transcarotid revascularisation for internal carotid artery stenosis: a systematic review and meta-analysis. Eur J Vasc Endovasc Surg 61:725-738

6. KashyapVS, Schneider PA, Foteh Metal (2020) Early outcomes in the ROADSTER 2 study of transcarotid artery revascularization in patients with significant carotid artery disease. Stroke 51:2620-2629

7. Kashyap VS, King AH, Foteh MI, Janko M, Jim J, Motaganahalli RL, Apple JM, Bose S, Kumins NH (2019) A multi-institutional analysis of transcarotid artery revascularization compared to carotid endarterectomy. JVasc Surg 70:123-129

8. Liang P, Soden P, Wyers MC, Malas MB, Nolan BW, Wang GJ, Powell RJ, Schermerhorn ML (2020) The role of transfemoral carotid artery stenting with proximal balloon occlusion embolic protection in the contemporary endovascular management of carotid artery stenosis. JVasc Surg 72:1701-1710

9. Malas MB, Dakour-Aridi H, Wang GJ, Kashyap VS, Motaganahalli RL, Eldrup-Jorgensen J, Cronenwett JL, Schermerhorn ML (2019) Transcarotid artery revascularization versus transfemoral carotid artery stenting in the Society for Vascular Surgery Vascular Quality Initiative. J Vasc Surg 69:92-103

10. Malas MB, Leal J, Kashyap V, Cambria RP, KwolekCJ, Criado E (2017) Technical aspects of transcarotid artery revascularization using the ENROUTE transcarotid neuroprotection and stent system. JVasc Surg 65:916-920

11. Naazie IN, Cui CL, Osaghae I, Murad MH, Schermerhorn M, Malas MB (2020) A systematic review and meta-analysis of transcarotid artery revascularization with dynamic flow reversal versus transfemoral carotid artery stenting and carotid endarterectomy. Ann Vasc Surg 69:426-436

12. Naazie IN, Magee GA, Mathlouthi A, Elsayed N, Dakour-Aridi H, Malas MB (2020) Primary mechanism of stroke reduction in transcarotid artery revascularization is dynamic flow reversal. J Vasc Surg.https://doi.org/10.1016/j.jvs.2020.10.082

13. Sagris M, Giannopoulos S, Giannopoulos $S$, Tzoumas A, Texakalidis P, Charisis N, Kokkinidis DG, Malgor RD, Mouawad NJ, Bakoyiannis C (2021) Transcervical carotid artery revascularization: a systematic review and meta-analysis of outcomes. J Vasc Surg. https://doi.org/10.1016/j.jvs.2021.03. 032
14. Schermerhorn ML, Liang $P$, Dakour-Aridi $H$, Kashyap VS, Wang GJ, Nolan BW, Cronenwett JL, Eldrup-Jorgensen J, Malas MB (2020) In-hospital outcomes of transcarotid artery revascularization and carotid endarterectomy in the Society for Vascular Surgery Vascular Quality Initiative. J Vasc Surg 71:87-95 